To APpear In The Astrophysical Journal LetTERS

Preprint typeset using LATEX style emulateapj v. 11/12/01

\title{
OPTICAL SPECTROPOLARIMETRY OF THE GRB 020813 AFTERGLOW
}

\author{
Aaron J. BArth ${ }^{1,2}$, Re'em SARi ${ }^{3}$, MARshall H. COHEN ${ }^{1}$, Robert W. Goodrich ${ }^{4}$, PAul A. Price ${ }^{1,5}$, \\ DereK W. FOX ${ }^{1}$, J. S. BloOM ${ }^{6}$, Alicia M. SODERBERG ${ }^{1}$, AND Shrinivas R. Kulkarni ${ }^{1}$ \\ To appear in The Astrophysical Journal Letters
}

\begin{abstract}
The optical afterglow of gamma-ray burst 020813 was observed for 3 hours with the LRIS spectropolarimeter at the Keck-I telescope, beginning 4.7 hours after the burst was detected by HETE-2. The spectrum reveals numerous metal absorption lines that we identify with two systems at $z=1.223$ and $z=1.255$. We also detect an [O II] $\lambda 3727$ emission line at $z=1.255$ and we identify this galaxy as the likely host of the GRB. After a correction for Galactic interstellar polarization, the optical afterglow has a linear polarization of $1.8-2.4 \%$ during $4.7-7.9$ hours after the burst. A measurement of $p=0.80 \% \pm 0.16 \%$ on the following night by Covino et al. demonstrates significant polarization variability over the next 14 hours. The lack of strong variability in the position angle of linear polarization indicates that the magnetic field in the jet is likely to be globally ordered rather than composed of a number of randomly oriented cells. Within the framework of afterglow models with collimated flows, the relatively low observed polarization suggests that the magnetic field components perpendicular and parallel to the shock front are only different by about $20 \%$.
\end{abstract}

Subject headings: gamma rays: bursts — polarization

\section{INTRODUCTION}

It is now generally assumed that the optical afterglows of gamma-ray bursts (GRBs) arise from synchrotron emission in the post-shocked gas of a relativistic blast wave (see, e.g., Piran 2000; Mészáros 2002). There is credible evidence that GRBs are strongly collimated or beamed explosions (e.g., Rhoads 1999; Sari, Piran, \& Halpern 1999; Frail et al. 2001). Synchrotron radiation is intrinsically polarized, and the synchrotron emission from a jet will have a net polarization if its axis does not coincide with the line of sight. A natural consequence of models for beamed afterglows is time-variable polarization, which will depend on the viewing geometry as well as the degree of orientation of the magnetic field (e.g., Medvedev \& Loeb 1999; Gruzinov 1999), up to a maximum polarization of 20\% (Ghisellini \& Lazzati 1999; Sari 1999). Optical imaging polarimetry has been performed for a handful of GRB afterglows to search for this signature. The results have been either upper limits (Hjorth et al. 1999; Covino et al. 2002a) or detections at $p \approx 1-3 \%$ (Covino et al. 1999; Wijers et al. 1999; Rol et al. 2000; Covino et al. 2002b), except for an observation of GRB 020405 by Bersier et al. (2002) that detected $9.9 \% \pm 1.3 \%$ polarization 1.3 days after the burst.

GRB 020813, a typical long-duration burst with a series of pulses, was detected by HETE-2 at 2:44 UT on 2002 August 13 (Villaseñor et al. 2002), and an associated optical transient (OT) was promptly identified (Fox, Blake, \& Price 2002). The rapid localization allowed us to begin spectropolarimetric observations less than 5 hours after the burst was initially detected. These are the first spectropolarimetric observations obtained for a GRB afterglow. Preliminary descriptions of this dataset were given by Price et al. (2002) and Barth et al. (2002).

\section{OBSERVATIONS AND REDUCTIONS}

The observations were obtained with the dual-beam LRIS spectropolarimeter (LRISp; Oke et al. 1995; Goodrich, Cohen, $\&$ Putney 1995) on the Keck-I telescope, and a slit width of $1^{\prime \prime}$. The addition of a new ultraviolet polarizing calibration filter to LRISp allowed us for the first time to take advantage of the full available wavelength range of LRISp using both the blue and red cameras. We used a 400 lines $\mathrm{mm}^{-1}$ grism on the blue side of the spectrograph and a 400 lines $\mathrm{mm}^{-1}$ grating on the red side, giving a wavelength scale of $1.09 \AA$ pixel $^{-1}$ over 3200 $5800 \AA$ in the blue and $1.86 \AA$ pixel $^{-1}$ over $5600-9400 \AA$ in the red. The red and blue beams were separated with a dichroic beamsplitter having 50\% transmission at $5700 \AA$.

We began exposures of the OT associated with GRB 020813 on 2002 August 13 at 7:23 UT, when its brightness was $V \approx 19$ mag. The total exposure time was 3 hours, broken into individual 15-minute exposures. Each pair of consecutive exposures gives one of the Stokes parameters $q$ (with the half-wave plate oriented at $0^{\circ}$ and $\left.45^{\circ}\right)$ or $u(22.5$ and 67.5$)$, and the 4 exposures taken together are used to determine the degree and position angle of linear polarization ( $p$ and $\theta$ ). We refer to these three sets of four exposures each as the "hour 1", "hour 2", and "hour 3" data. The airmass was 1.3-1.5 during the observations, and before starting each hour's exposures the spectrograph was rotated to place the slit at the parallactic angle for the midpoint of the hour. Seeing was $\sim 0 !^{\prime \prime} 7$, and there were intermittent thin clouds during the observations.

As a probe of the Galactic interstellar polarization (ISP), we also observed the A2V star HD 187330, which is located 64 .7 from the line of sight to the GRB. Its apparent magnitude $(V=9.22 \mathrm{mag})$ implies a distance of $\sim 350 \mathrm{pc}$; this is sufficiently distant to probe the majority of the Galactic ISP for a

\footnotetext{
${ }^{1}$ Astronomy Department, 105-24 Caltech, Pasadena, CA 91125; barth@astro.caltech.edu

${ }^{2}$ Hubble Fellow

${ }^{3}$ Theoretical Astrophysics, 130-33 Caltech, Pasadena, CA 91125

${ }^{4}$ W. M. Keck Observatory, 65-1120 Mamalahoa Highway, Kamuela, HI 96743

${ }^{5}$ Research School of Astronomy and Astrophysics, Mount Stromlo Observatory, via Cotter Road, Weston, ACT 2611, Australia

${ }^{6}$ Harvard-Smithsonian Center for Astrophyics, 60 Garden St., Cambridge, MA 02138
} 
Galactic latitude of $-21^{\circ}$ (Tran 1995). Distant A-type stars are useful as probes of ISP because of their brightness and low intrinsic polarization, although ideally a smaller angular separation from the OT would be desirable.

The data were reduced using standard techniques as described by Miller, Robinson, \& Goodrich (1988). The zeropoint of $\theta$ was calibrated with the polarized standard star $\mathrm{BD}+59^{\circ} 389$ (Schmidt et al. 1992). For the unpolarized standard star HD 14069 we found $p \leq 0.1 \%$ across the entire optical spectrum.

\section{CONTINUUM AND ABSORPTION LINES}

Figure 1 displays the total flux spectrum of the OT from all exposures combined. Numerous metal absorption lines are superposed on a featureless continuum, as is typical for GRB afterglow spectra (e.g., Metzger et al. 1997; Kulkarni et al. 1999; Vreeswijk et al. 2001; Jha et al. 2001). Two distinct absorption systems are present in the spectrum (Table 2). The individual absorption lines in the higher-redshift system have a mean $z=1.255$. The second system has weaker absorption lines and a mean redshift of 1.223. Both systems show lines of Si II $\lambda 1527$, C IV $\lambda \lambda 1548,1551, \mathrm{Al}$ III $\lambda 1671, \mathrm{Mg}$ II $\lambda \lambda 2796,2804$, and Mg I $\lambda 2853$, as well as several Fe II lines including $\lambda \lambda 2587,2600$. Additional features from the $z=1.255$ system include lines of Al III, Mn II, Mg I, Zn II, and Cr II. One emission line is also present in the spectrum, centered at $8404.0 \AA$ and consistent with [O II] $\lambda 3727$ at $z=1.255$. We conclude that the $z=1.255$ system is the likely host of the GRB, since it is the highestredshift absorption system detected and its [O II] emission line is an indicator of star-forming activity.

Ionization of the surrounding interstellar medium (ISM) by the GRB could cause temporal variations in the equivalent widths of interstellar absorption lines within the host galaxy (Perna \& Loeb 1998), and we have measured the equivalent widths of the absorption lines in the hour 1, 2, and 3 spectra separately to search for any variations. The differences in equivalent widths are consistent with the level of measurement uncertainty, and we conclude that no variability is detected. Searches for ultraviolet absorption-line variability in other GRBs have so far yielded null results as well (Vreeswijk et al. 2001; Mirabal et al. 2002).

The continuum has a power-law slope of $\beta=-1.06 \pm 0.01$ (for $f_{\nu} \propto \nu^{\beta}$ ) on the red side. On the blue side, we find a steeper slope of $\beta=-1.25 \pm 0.03$. This apparent steepening toward the blue is affected by atmospheric dispersion and is sensitive to the centering of the OT within the slit, as well as reddening, so the red-side value is a more reliable measurement of the intrinsic slope. Levan et al. (2002) reported a shallower optical slope of $\beta=-0.8$ approximately 98 hours after the burst.

\section{POLARIZATION}

Polarization was measured over five wavelength bins: $3500-$ $4500,4500-5500,5800-6800,6800-7800$, and $7800-8800$ $\AA$. All measurements were performed using the $q$ and $u$ spectra and their associated error spectra, with the results converted to $p$ and $\theta$ as the final step. We observed the same three polarized standard stars $\left(\mathrm{BD}+59^{\circ} 389\right.$, HD 19820, and HD 155528) during both nights of our observing run (August 12 and 13 UT). For all three stars and five wavelength bins, the RMS difference in the polarization measured from the two nights' observations is only $0.04 \%$. The polarized standard stars also have essentially constant $\theta$ as a function of wavelength (to within $\pm 1^{\circ}$ ), and agreement to within $1^{\circ}$ between the two nights of observations. This demonstrates the excellent stability of the instrument.

The raw measurements for the OT yield $p=2.3-3.1 \%$ with $\theta=153^{\circ}-162^{\circ}$, but a portion of this polarization is caused by transmission through regions of aligned dust grains in the Galactic ISM. The line of sight to GRB 020813 has a Galactic reddening of $E(B-V)=0.111 \mathrm{mag}$ (Schlegel, Finkbeiner, $\&$ Davis 1998). Along most Galactic lines of sight, the maximum interstellar polarization observed is $9 \%$ per magnitude of $E(B-V)$ (Serkowski, Mathewson, \& Ford 1975). The ISP probe star has $p=0.67 \% \pm 0.01 \%$ at $\theta=167.0 \pm 0.3$ over $5800-6800 \AA$, falling comfortably within the expected range, and its $p(\lambda)$ spectrum is consistent with a Serkowski interstellar polarization curve. To correct the polarization measurements of the OT, we fitted a low-order polynomial to the $q$ and $u$ spectra of the ISP star and subtracted the result from the Stokes parameters of the OT. The ISP-corrected measurements for the OT are listed in Table 1; random uncertainties due to photon statistics and detector read noise are at the level of $0.1 \%$ in $p$ and $1-2^{\circ}$ in $\theta$. The standard star observations suggest that any additional systematic uncertainties should not be time-variable, and are likely to be $\lesssim 0.1 \%$ in $p$ and $\lesssim 1^{\circ}$ in $\theta$.

The ISP-corrected polarization longward of $5600 \AA$ is nearly constant during the 3 hours at 1.9-2.2\%. On the blue side, however, the polarization drops from $2.4 \% \pm 0.1 \%$ during hour 1 to $1.8 \% \pm 0.1 \%$ in hour 3 . This is formally a $4 \sigma$ detection of polarization variability, but it is somewhat surprising to find significant variations in $p$ only on the blue side. As a check on this measurement, the reductions were repeated with different extraction and background windows, and repeated again using entirely different reduction software; in all cases the results were consistent with the original measurements to within $0.1 \%$ in $p$. We conclude that the variability in $p$ on the blue side is likely to be genuine, although we caution against over-interpretation of this result since the measurement is not repeatable.

Imaging polarimetry obtained on the following night at the VLT by Covino et al. (2002c) demonstrates a much more dramatic level of variability in $p$ over the next 14 hours. Covino et al. find a raw polarization of $p=1.17 \% \pm 0.16 \%$ at $\theta=$ $158^{\circ} \pm 4^{\circ}$, and ISP-corrected values of $p=0.80 \% \pm 0.16 \%$ at $\theta=144^{\circ} \pm 6^{\circ} .7$ If we apply our ISP correction to their raw measurement we obtain $p=0.57 \%$ at $\theta=147^{\circ}$. The change in $p$ between our hour 3 data and the VLT observation is significant at the $7 \sigma$ level. This large drop in $p$ demonstrates that most of the polarization we detect in the Keck data must be intrinsic to the OT itself, and not merely the result of transmission through dust within the host galaxy or the intervening galaxy at $z=1.223$.

The ISP-corrected polarization detected by Covino et al. (2002c) is sufficiently low that in principle it could arise from the interstellar medium in the host galaxy or perhaps even in the intervening $z=1.223$ system, although dust grains have a low polarization efficiency in the ultraviolet (Martin, Clayton, $\&$ Wolff 1999). Furthermore, if GRBs result from the death of massive stars, one can expect many GRBs to occur in dusty and gas-rich star-forming regions, and in extreme cases Type II supernovae can have optical ISP of several percent from the host galaxy (Leonard et al. 2002). However, Chandra observations have indicated a low absorbing column of $N_{\mathrm{H}}=7 \times 10^{20} \mathrm{~cm}^{-2}$

\footnotetext{
${ }^{7}$ Covino et al. (2002c) use an ISP correction derived from stars in the same imaging field as the OT. Their $V$-band ISP correction is $p=0.59 \%$ at $\theta=178^{\circ}$, in reasonable agreement with the polarization we measure for HD 187330.
} 
that is consistent with the expected Galactic absorption along the line of sight (Vanderspek et al. 2002). Also, the lack of any large change in $\theta$ accompanying the variation in $p$ suggests an intrinsic origin for the majority of the detected polarization. Detection of a continued late-time decline in $p$ would provide a definitive test.

\section{DISCUSSION}

Our observations, combined with those of Covino et al. (2002c), clearly show variability in the degree of polarization, with a nearly constant position angle. This already places strong constraints on the polarization models. Random effects, such as emission from a small number of randomly oriented magnetic field cells (Gruzinov \& Waxman 1999) or amplification of the emission from one such cell by microlensing (Loeb $\&$ Perna 1998), would predict that $p$ and $\theta$ should change on the same timescale.

In principle, the jet geometry, together with an assumption of some level of orientation of the amplified magnetic field with respect to the shock, could predict the degree of polarization as function of time. Models based on this scenario (Ghisellini \& Lazzati 1999; Sari 1999) show that the maximum degree of polarization occurs around the time of the jet break $\left(t_{\mathrm{jet}}\right)$, when the opening angle of the jet is equal to the inverse Lorentz factor. However, at that time the emission is observable from a significant fraction of the jet. This introduces order-unity uncertainties in the polarization predictions since the energy distribution profile as function of distance from the axis of the jet is unknown. In addition, a detailed description of the hydrodynamic evolution is not yet available; only the asymptotic scalings, well before and well after $t_{\text {jet }}$, are understood. Thus, it is not possible to make detailed, quantitative comparisons of polarization models with observations.

Despite these uncertainties, we can make general comparisons of the observations with the model proposed by Sari (1999), which predicts sharp features in the polarization light curves, although more realistic assumptions on the hydrodynamics of jet spreading, and on the energy distribution within the initial jet, are expected to produce smoother features than those predicted by this model. This model has three parameters. The line-of-sight offset to the jet determines the shape of the curve; $t_{\text {jet }}$ sets the overall timescale; and an overall scale factor for $p$ is set by a function of the ratio of the magnetic field components parallel to and perpendicular to the shock front, which is assumed to be constant. We denote the offset in units of the opening angle of the jet by $0<\phi<1$. (For $\phi>1$ the jet is not visible initially, corresponding to an "orphan" afterglow.) Small offsets give rise to a single polarization peak, while large offsets yield three peaks, where the middle one has position angle perpendicular to the other two. More realistic jet models may smooth the curve and eliminate this middle peak. We note that the model of Ghisellini \& Lazzati (1999) predicts only two peaks since their calculations did not include the spreading of the jet.

Figure 2 demonstrates that the jet model parameters can be chosen so as to find at least a qualitative agreement with the data. In the figure, our measurements for the 5800-6800 polarization, and the $V$-band measurement from Covino et al. (2002c), are compared with two sample models. Initial analysis of the optical light curve suggested $t_{\mathrm{jet}} \approx 3.5-5$ hours (Bloom, Fox, \& Hunt 2002; Gladders \& Hall 2002), although subsequent analysis (to be presented elsewhere) indicates that the light curves may be consistent with a later value of $t_{\text {jet }} \approx 10-11$ hours. Given this uncertainty in deriving $t_{\text {jet }}$ from the photometric data, our approach is to determine whether there are any values of $t_{\mathrm{jet}}$ that produce polarization light curves consistent with the observations. The first model has a small offset $\phi=0.3$ and predicts one peak of polarization. (Models with $0<\phi \lesssim 0.35$ would give similar results.) This requires $t_{\text {jet }}$ to occur during the Keck observations, at $\sim 6$ hours. However, this model somewhat underpredicts the decay of polarization between our measurements and those of Covino et al. A better fit is obtained for a medium-offset model with $\phi=0.55$ and $t_{\text {jet }} \approx 16$ hours, where the polarization drops to zero, or perhaps even rotates the position angle by $90^{\circ}$, between the Keck and VLT observations. Under both interpretations, the overall level of polarization is less than the maximal possible level of $\sim 20 \%$. This implies that the magnetic field perpendicular to the shock differs only by $15-20 \%$ from that parallel to the shock front. Models with other values of $\phi$ have difficulty matching both the near-constancy of $p$ during the Keck observations (for $\lambda>5800$ $\AA$ ) and the large change in $p$ detected by Covino et al. (2002c).

The polarization behavior of GRB 020813 appears rather similar to that of GRB 990712, which varied between $p=3.0 \%$ at 0.4 days after the burst and $1.2 \%$ at $t=0.8$ days, at nearly constant position angle (Rol et al. 2000). We note that our models with $\phi=0.3$ or 0.55 could also be nearly consistent with the GRB 990712 data within its uncertainties. Still, as noted by Rol et al., the lack of clear evidence for a jet break for that event casts some doubt on this interpretation.

Finally, we mention that the hints of wavelength dependence to the polarization of GRB 020813, which appear to be timevariable, can not be accommodated by these models. A spectral break passing through the optical band at the time of our three measurements could produce such a signature, but there is no evidence for such a break.

To date, polarimetric observations of GRB afterglows have had rather sparse temporal coverage. Further progress will require continuous polarization monitoring of bright afterglows, initiated as early as possible and extending well beyond $t_{\text {jet }}$. Also, our data highlight the need for color information in future polarization measurements. Obtaining such observations would pose some logistical difficulties, but the potential for improved understanding of the physical structure of the synchrotron jets from GRBs would make this effort extremely worthwhile.

Research by A.J.B. is supported by NASA through Hubble Fellowship grant \#HST-HF-01134.01-A awarded by STScI, which is operated by AURA, Inc., for NASA, under contract NAS 5-26555. R.S. thanks the Sherman Fairchild foundation and a NASA ATP grant for support. Data presented herein were obtained at the W.M. Keck Observatory, which is operated as a scientific partnership among Caltech, the University of California, and NASA. The Observatory was made possible by the generous financial support of the W.M. Keck Foundation. The authors wish to recognize and acknowledge the very significant cultural role and reverence that the summit of Mauna Kea has always had within the indigenous Hawaiian community. 


\section{REFERENCES}

Barth, A. J., Cohen, M. H., Goodrich, R. W., Price, P. A., Bloom, J. S., \& Fox, D. W. 2002, GCN Circ. 1477

Bersier, D., et al. 2002, ApJ, submitted

Bloom, J. S., Fox, D. W., \& Hunt, M. P. 2002, GCN Circ. 1476

Covino, S., et al. 1999, A\&A, 348, L1

Covino, S., et al. 2002a, A\&A, 392, 865

Covino, S., et al. 2002b, A\&A, in press

Covino, S., et al. 2002c, GCN Circ. 1498

Fox, D. W., Blake, C., \& Price, P. 2002, GCN Circ. 1470

Frail, D. A., et al. 2001, ApJ, 562, L55

Ghisellini, G., \& Lazzati, D. 1999, MNRAS, 309, L7

Gladders, M., \& Hall, P. 2002, GCN Circ. 1514

Goodrich, R. W., Cohen, M. H., \& Putney, A. 1995, PASP, 107, 179

Gruzinov, A. 1999, ApJ, 525, L29

Gruzinov, A., \& Waxman, E. 1999, ApJ, 511, 852

Hjorth, J., et al. 1999, Science, 283, 2073

Jha, S., et al. 2001, ApJ, 554, L155

Kulkarni, S. R., et al. 1999, Nature, 398, 389

Leonard, D. C., Filippenko, A. V., Chornock, R., \& Li, W. 2002, AJ, 124, 2506

Levan, A. J., Fruchter, A. S., Burud, I., \& Rhoads, J. E. 2002, GCN Circ. 1518

Loeb, A., \& Perna, R. 1998, ApJ, 495, 597

Martin, P. G., Clayton, G. C., \& Wolff, M. J. 1999, ApJ, 510, 905

Medvedev, M. V., \& Loeb, A. 1999, ApJ, 526, 697

Mészáros, P. 2002, ARA\&A, 40, 137
Metzger, M. R., Djorgovski, S. G., Kulkarni, S. R., Steidel, C. C., Adelberger, K. L., Frail, D. A., Costa, E., \& Frontera, F. 1997, Nature, 387, 878

Miller, J. S., Robinson, L. B., \& Goodrich, R. W. 1988, in Instrumentation for Ground-Based Astronomy, ed. L. B. Robinson (New York: Springer-Verlag), 157

Mirabal, N., et al. 2002, ApJ, 578, 818

Oke, J. B., et al. 1995, PASP, 107, 375

Piran, T. 2000, Phys. Rep., 333, 529

Perna, R., \& Loeb, A. 1998, ApJ, 501, 467

Price, P. A., Bloom, J. S., Goodrich, R. W., Barth, A. J., Cohen, M. H., \& Fox, D. W. 2002, GCN Circ. 1475

Rhoads, J. E. 1999, ApJ, 525, 737

Rol, E., et al. 2000, ApJ, 544, 707

Sari, R. 1999, ApJ, 524, L43

Sari, R., Piran, T., \& Halpern, J. P. 1999, ApJ, 519, L17

Schmidt, G. D., Elston, R., \& Lupie, O. L. 1992, AJ, 104, 1563

Schlegel, D. J., Finkbeiner, D. P., \& Davis, M. 1998, ApJ, 500, 525

Serkowski, K., Mathewson, D. S., \& Ford, V. L. 1975, ApJ, 196, 261

Tran, H. D. 1995, ApJ, 440, 565

Vanderspek, R., Marshall, H. L., Ford, P. G., \& Ricker, G. R. 2002, GCN Circ. 1504

Villaseñor, J., et al. 2002, GCN Circ. 1471

Vreeswijk, P. M., et al. 2001, ApJ, 546, 672

Wijers, R. A. M. J., et al. 1999, ApJ, 523, L33

TABLE 1

Polarization MeAsurements

\begin{tabular}{cccc}
\hline \hline Hour & $\begin{array}{c}\text { Wavelength } \\
(\AA)\end{array}$ & $\begin{array}{c}p \\
(\%)\end{array}$ & $\begin{array}{c}\theta \\
\left({ }^{\circ}\right)\end{array}$ \\
\hline 1 & $3500-4500$ & $2.4 \pm 0.1$ & $161 \pm 1$ \\
& $4500-5500$ & $2.4 \pm 0.1$ & $159 \pm 1$ \\
& $5800-6800$ & $2.1 \pm 0.1$ & $158 \pm 1$ \\
& $6800-7800$ & $2.1 \pm 0.1$ & $155 \pm 1$ \\
& $7800-8800$ & $2.1 \pm 0.1$ & $155 \pm 1$ \\
& $3500-4500$ & $1.8 \pm 0.1$ & $160 \pm 1$ \\
& $4500-5500$ & $2.0 \pm 0.1$ & $155 \pm 1$ \\
& $5800-6800$ & $2.0 \pm 0.1$ & $151 \pm 1$ \\
& $6800-7800$ & $2.0 \pm 0.1$ & $150 \pm 1$ \\
& $7800-8800$ & $2.1 \pm 0.1$ & $151 \pm 2$ \\
& $3500-4500$ & $1.8 \pm 0.1$ & $153 \pm 1$ \\
& $4500-5500$ & $1.8 \pm 0.1$ & $149 \pm 1$ \\
& $5800-6800$ & $2.1 \pm 0.1$ & $156 \pm 1$ \\
& $6800-7800$ & $2.2 \pm 0.1$ & $153 \pm 1$ \\
& $7800-8800$ & $1.9 \pm 0.1$ & $149 \pm 1$ \\
& & &
\end{tabular}

Note. - The results given above have been corrected for ISP using observations of the A2V star HD 187330 , as described in the text. The midpoints of the hour 1,2 , and 3 observations were at 7:54, 9:01, and 10:06 UT, respectively. 
TABLE 2

ABSORPTION LINES IN THE GRB 020813 SPECTRUM

\begin{tabular}{|c|c|c|c|c|}
\hline $\begin{array}{c}\lambda_{\text {helio }} \\
(\AA)\end{array}$ & Ion & $\begin{array}{l}\lambda_{\text {rest }} \\
(\AA)\end{array}$ & $z$ & $\begin{array}{c}W_{\lambda}(\text { rest }) \\
(\AA)\end{array}$ \\
\hline 3393.8 & Si II & 1526.7 & 1.223 & $0.46 \pm 0.12$ \\
\hline 3442.3 & $\begin{array}{l}\text { C IV blend } \\
+\mathrm{Si} \text { II }\end{array}$ & $\begin{array}{c}1548.2,1550.8 \\
1526.7\end{array}$ & $\begin{array}{l}1.222 \\
1.255\end{array}$ & $2.05 \pm 0.06$ \\
\hline 3456.2 & $\ldots$ & $\ldots$ & $\ldots$ & $1.71 \pm 0.18^{\mathrm{a}}$ \\
\hline 3489.4 & C IV & 1548.2 & 1.254 & $1.53 \pm 0.05$ \\
\hline 3495.1 & C IV & 1550.8 & 1.254 & $1.31 \pm 0.06$ \\
\hline 3626.3 & $\mathrm{Fe}$ II & 1608.5 & 1.254 & $1.05 \pm 0.07$ \\
\hline 3714.6 & Al II & 1670.8 & 1.223 & $0.68 \pm 0.04$ \\
\hline 3766.9 & Al II & 1670.8 & 1.255 & $1.48 \pm 0.04$ \\
\hline 4077.5 & Si II & 1808.0 & 1.255 & $0.71 \pm 0.02$ \\
\hline 4182.6 & Al III & 1854.7 & 1.255 & $0.91 \pm 0.02$ \\
\hline 4200.9 & Al III & 1862.8 & 1.255 & $0.58 \pm 0.03$ \\
\hline 4569.0 & Mg I, Zn II , Cr II & $2026.5,2026.1,2026.3$ & 1.255 & $0.61 \pm 0.03$ \\
\hline 4636.3 & Cr II & 2056.3 & 1.255 & $0.30 \pm 0.03$ \\
\hline 4650.6 & Cr II, Zn II & $2062.2,2062.7$ & 1.255 & $0.44 \pm 0.03$ \\
\hline 4658.5 & Cr II & 2066.2 & 1.255 & $0.17 \pm 0.03$ \\
\hline 4884.2 & $\ldots$ & $\ldots$ & $\ldots$ & $0.58 \pm 0.07^{\mathrm{a}}$ \\
\hline 4998.5 & $\mathrm{Fe}$ II & 2249.9 & 1.222 & $0.35 \pm 0.03$ \\
\hline 5072.5 & $\mathrm{Fe}$ II & 2249.9 & 1.255 & $0.23 \pm 0.02$ \\
\hline 5097.3 & $\mathrm{Fe}$ II & 2260.8 & 1.255 & $0.35 \pm 0.03$ \\
\hline 5211.9 & $\mathrm{Fe}$ II & 2344.2 & 1.223 & $0.50 \pm 0.03$ \\
\hline 5223.2 & $\ldots$ & $\ldots$ & $\ldots$ & $0.51 \pm 0.08^{\mathrm{a}}$ \\
\hline 5285.3 & $\mathrm{Fe}$ II & 2344.2 & 1.255 & $2.00 \pm 0.02$ \\
\hline 5297.6 & $\mathrm{Fe}$ II & 2382.8 & 1.223 & $1.01 \pm 0.03$ \\
\hline 5354.1 & $\mathrm{Fe}$ II & 2374.5 & 1.255 & $1.42 \pm 0.03$ \\
\hline 5372.5 & $\mathrm{Fe}$ II & 2382.8 & 1.255 & $2.10 \pm 0.03$ \\
\hline 5402.3 & $\ldots$ & $\ldots$ & $\ldots$ & $0.64 \pm 0.07^{\mathrm{a}}$ \\
\hline 5752.1 & $\mathrm{Fe}$ II & 2586.7 & 1.224 & $0.45 \pm 0.04$ \\
\hline 5782.0 & $\mathrm{Fe}$ II & 2600.2 & 1.224 & $0.97 \pm 0.03$ \\
\hline 5811.4 & Mn II & 2576.9 & 1.255 & $0.66 \pm 0.03$ \\
\hline 5833.1 & $\mathrm{Fe}$ II & 2586.7 & 1.255 & $1.92 \pm 0.04$ \\
\hline 5851.4 & Mn II & 2594.5 & 1.255 & $0.57 \pm 0.03$ \\
\hline 5863.4 & $\mathrm{Fe} I I$ & 2600.2 & 1.255 & $2.31 \pm 0.04$ \\
\hline 5877.4 & Mn II & 2606.5 & 1.255 & $0.37 \pm 0.03$ \\
\hline 5882.8 & $\ldots$ & $\ldots$ & $\ldots$ & $0.89 \pm 0.11^{\mathrm{a}}$ \\
\hline 5892.4 & $\ldots$ & $\ldots$ & $\ldots$ & $0.71 \pm 0.15^{\mathrm{a}}$ \\
\hline 6218.4 & Mg II & 2796.4 & 1.224 & $1.67 \pm 0.02$ \\
\hline 6234.3 & $\mathrm{Mg}$ II & 2803.5 & 1.224 & $1.52 \pm 0.02$ \\
\hline 6305.7 & $\mathrm{Mg}$ II & 2796.4 & 1.255 & $2.94 \pm 0.02$ \\
\hline 6321.9 & $\mathrm{Mg}$ II & 2803.5 & 1.255 & $2.82 \pm 0.02$ \\
\hline 6344.4 & $\mathrm{Mg} \mathrm{I}$ & 2853.0 & 1.224 & $0.36 \pm 0.02$ \\
\hline 6433.4 & $\mathrm{Mg} \mathrm{I}$ & 2853.0 & 1.255 & $1.61 \pm 0.02$ \\
\hline 8872.6 & $\mathrm{Ca}$ II & 3934.8 & 1.255 & $2.07 \pm 0.05$ \\
\hline 8951.7 & Ca II & 3969.6 & 1.255 & $1.56 \pm 0.07$ \\
\hline
\end{tabular}

Note. - Observed wavelengths are heliocentric, and converted to vacuum. Rest wavelengths are given as vacuum wavelengths. Absorption-line equivalent widths $\left(W_{\lambda}\right)$ and uncertainties were measured with Gaussian fits using the IRAF SPLOT task, and have been transformed to the rest frame.

${ }^{a}$ For unidentified absorption lines, equivalent widths are given in the observed frame. 


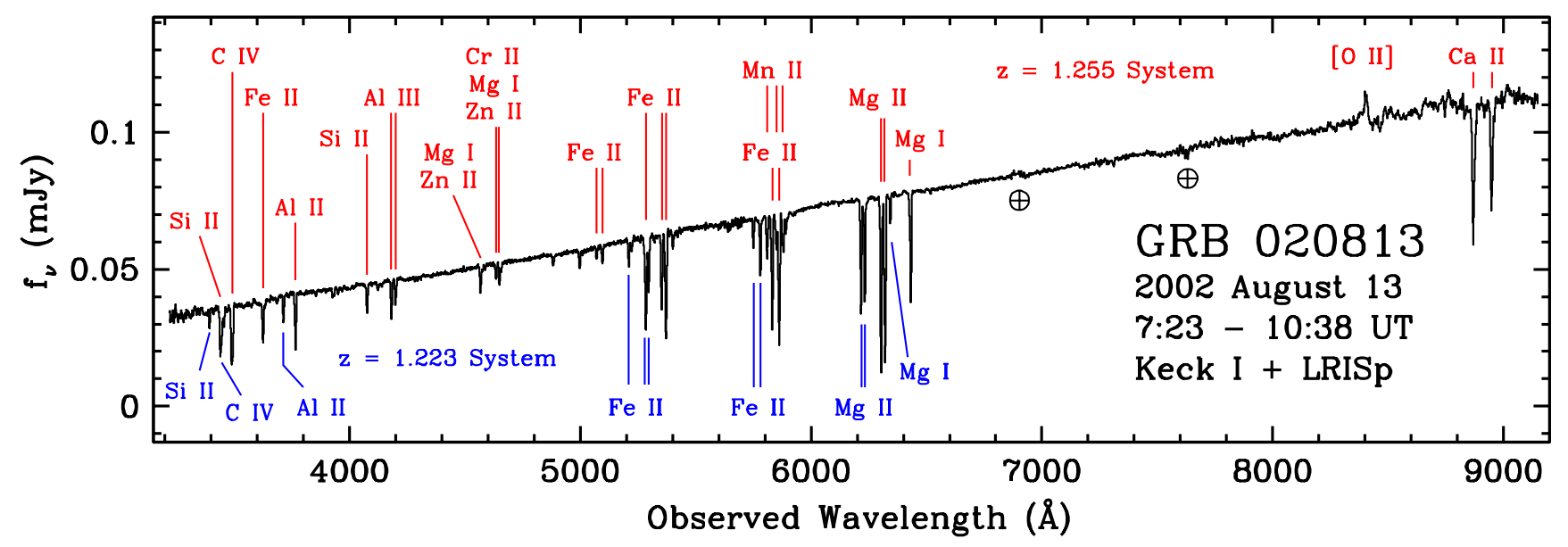

FIG. 1.- Total flux spectrum of GRB 020813, from all LRISp exposures combined. Absorption features from the $z=1.255$ system are labelled above the spectrum, and features from the $z=1.223$ system are labelled below. Residuals from the removal of the telluric A and B bands are marked with $\oplus$.

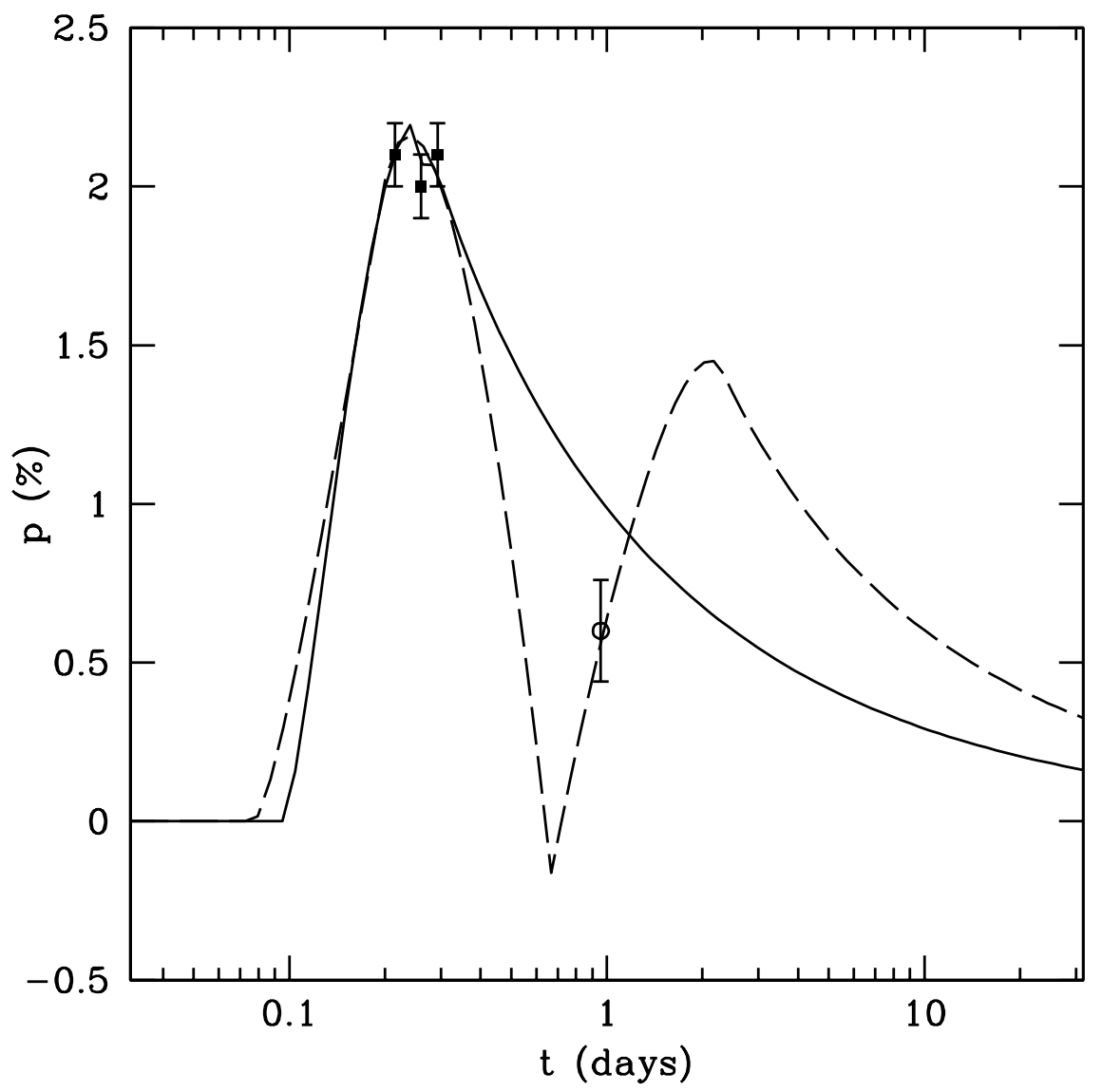

FIG. 2.- Polarization behavior of GRB 020813 compared with model calculations. Squares denote the Keck observations in the $5800-6800 \AA$ band, and the open circle is the VLT measurement from Covino et al. (2002c). The solid curve is the model with $\phi=0.3$ and $t_{\text {jet }}=6$ hours, and the dashed curve is the model with $\phi=0.55$ and $t_{\text {jet }}=16$ hours. Negative polarizations in this plot indicate a rotation of $90^{\circ}$ in the position angle relative to positive polarizations. For consistency, we have applied our ISP correction to the VLT data point. 